http://dx.doi.org/10.35381/r.k.v6i4.1507

\title{
Narrativa digital y gestión educativa: Estrategia para la motivación al quehacer docente
}

Digital storytelling and education management: A strategy for teacher's motivation at work

\author{
Christian Javier Bedoya-Merchán \\ chrisbedoyam@gmail.com \\ Universidad Católica de Cuenca, Cuenca \\ Ecuador \\ https://orcid.org/0000-0002-9273-7952 \\ Santiago Arturo Moscoso-Bernal \\ smoscoso@ucacue.edu.ec \\ Universidad Católica de Cuenca, Cuenca \\ Ecuador \\ https://orcid.org/0000-0002-7647-1111 \\ Ernesto Andrés Hermann Acosta \\ ernesto.hermann@ucacue.edu.ec \\ Universidad Católica de Cuenca, Quito \\ Ecuador \\ https://orcid.org/0000-0001-9068-0692
}

Recepción: 30 de agosto 2021

Revisado: 20 de septiembre 2021

Aprobación: 15 de noviembre 2021

Publicación: 01 de diciembre 2021 
Revista Arbitrada Interdisciplinaria KOINONIA

Año VI. Vol VI. N4. Edición Especial: Educación III. 2021

Hecho el depósito de Ley: FA2016000010 ISSN: 2542-3088

FUNDACIÓN KOINONIA (F.K). Santa Ana de Coro. Venezuela.

Jener Fabricio Chica-Correa; María Isabel Álvarez-Lozano; Claudio Fernando Guevara-Vizcaíno

\title{
RESUMEN
}

Se sobreentiende que el estado emocional, psicológico, nivel de satisfacción y actitud influye directamente en su quehacer y también en sus estudiantes. El propósito de este trabajo es analizar de qué manera el uso de la narrativa digital puede aportar a la motivación de los docentes desde la gestión educativa y para fortalecimiento institucional. El abordaje se lo ha realizado por medio de un enfoque cualitativo. El levantamiento de datos se ejecutó con grupo focal y su respectivo recurso instrumental como es la entrevista. Los resultados obtenidos demuestran la importancia de motivar a los docentes en su labor de manera eficiente, efectiva y continua con el uso de la narrativa digital como estrategia y que como consecuencia aseguraría que la gestión directiva se vea fortalecida.

Descriptores: Recursos educacionales; medios electrónicos; digitalización. (Palabras tomadas del Tesauro UNESCO).

\begin{abstract}
It is understood that the emotional, psychological state, level of satisfaction and attitude directly influence their work and also their students. The purpose of this work is to analyze how the use of digital narrative can contribute to the motivation of teachers from educational management and for institutional strengthening. The approach has been carried out through a qualitative approach. The data collection was carried out with a focus group and its respective instrumental resource, such as the interview. The results obtained demonstrate the importance of motivating teachers in their work efficiently, effectively and continuously with the use of digital narrative as a strategy and that as a consequence it would ensure that directive management is strengthened.
\end{abstract}

Descriptors: Educational resources; electronic media; digitization. (Words taken from the UNESCO Thesaurus). 


\section{INTRODUCCIÓN}

La dinámica educativa presenta cambios continuos y constantes, esto implica que los niveles de exigencia al personal docente, en las escuelas y colegios, sea una comúnii y compleja realidad a la que el sistema los expone por el conjunto de actividades y responsabilidades propias de la labor del profesorado, por ello, se entiende que a la hora de enseñar es necesario considerar todas las áreas contextuales de la persona que ejerce la enseñanza, es decir su estado emocional, psicológico, etc.

En el marco legal educativo, en el artículo 10 de los derechos de los docentes, literal a, la Ley Orgánica de Educación Intercultural [LOEI], 2011), menciona la importancia de que el profesorado pueda "Acceder gratuitamente a procesos de desarrollo profesional, capacitación, actualización, formación continua, mejoramiento pedagógico y académicos en todos los niveles y modalidades..." Así como también en el artículo 11 literal k, indica que es obligación de los maestros "Procurar una formación académica continua y permanente a lo largo de su vida, aprovechando oportunidades de desarrollo profesional existentes". Por lo tanto, la normativa deja clara la importancia de la formación integral [profesional, académica, personal y emocional] del cuerpo docente.

Por otra parte, el artículo 44 en las atribuciones del rector, en el Reglamento General a la (Ley Orgánica de Educación Intercultural [R-LOEI], 2016), plantea que la autoridad debe "Dirigir y controlar la implementación eficiente de programas académicos, y el cumplimiento del proceso de diseño y ejecución de los diferentes planes o proyectos institucionales, así como participar en su evaluación permanente y proponer ajustes". Razón por la que es responsabilidad del directivo, el planteamiento de estrategias institucionales de manera asertiva y pertinente en su gestión para mejorar la labor de los profesores y su impacto en el proceso de enseñanza.

Por lo antes mencionado, es evidente que el personal de enseñanza tiene que ser capacitado, actualizado, formado y especialmente motivado de manera continua para el ejercicio de sus funciones, mismas que deben caracterizarse por la calidad y calidez. En 
esta misma línea (Katz \& Shahar, 2015), aseguran que la motivación en la labor docente y el entusiasmo demostrado en sus actividades tienen un alto impacto en la motivación de los estudiantes y por ende en el aprendizaje. Por ende, los estados emocionales, psicológicos, grados de satisfacción y actitudes del maestro, influirán directamente en su quehacer pedagógico y los resultados académicos de sus aprendices.

La gestión docente es fundamental para el alcance de los objetivos institucionales, y el soporte de la gestión directiva es trascendental, para efectivizar los resultados esperados.

Por ello, los directivos deben ser conscientes que su trabajo está sesgado por el cúmulo de actitudes, situaciones afectivas, socio-relacionales y emocionales que como individuos experimentamos en el ejercicio de la profesión. Las exigencias propias de la labor educativa pueden terminar fatigando al personal con el llamado síndrome de burnout o síndrome del trabajador quemado.

En su investigación (Vicente-de-Vera-García \& Gabari-Gambarte, 2019), afirman que el burnout es un estado de cúmulos continuos de estrés en el área laboral, como resultado de las dinámicas propias del contexto del trabajo o fruto de las interrelaciones con los colegas o personas con quienes se labora. En el medio de la educación, se da un declive del contingente emocional del docente fruto de las exigencias del cargo, cantidades de estudiantes, exceso de trabajo académico, actividades administrativas, horarios y burocracia. Razón suficiente para comprender lo importante de la motivación continua y la contención emocional al trabajo de los maestros.

La gestión directiva tiene que considerar, no solo los resultados académicos y la efectiva aplicación de estrategias en el proceso de inter-aprendizaje, sino fortalecer su labor por medio del acompañamiento y la contención emocional al profesorado de una manera creativa, asertiva y colaborativa, con acciones que motiven a ejecutar una labor humana, consciente y motivada de manera intrínseca. Si se considera que existen herramientas que permiten un mayor alcance, gracias a la ubicuidad tecnológica y las posibilidades de 
asincronismo para el seguimiento al personal, entonces el uso y la generación de narrativa digital podrían enriquecer dicho proceso.

El presente trabajo pretende analizar de qué manera el uso de la narrativa digital, puede aportar a la motivación de docentes desde la gestión directiva y para fortalecimiento en la Unidad Educativa Unitec Discovery, ubicada en la ciudad de Cuenca - Ecuador. Este logro será posible en cuatro pasos: a) argumentar desde la teoría el uso de la narrativa digital y su influencia en la motivación de los docentes, b) identificar las necesidades de motivación en los docentes; y c) proponer la construcción continua de más productos para el intercambio de experiencias docentes para el fortalecimiento motivacional de la labor docente.

\section{Referencial teórico}

Para la producción del presente epígrafe se han examinado artículos científicos de las bases de datos Scopus, Redalyc y ReHuSo entre los años 2015 - 2021 a nivel global, regional y local. La estrategia utilizada para la organización y estructura, como lo plantea, (Calderón et al. 2014), implica la compilación de diferentes fuentes científicas relacionadas con la disciplina que se investiga para obtener los aportes de la problemática, la temática y su respectiva contextualización.

En el ámbito global, hay estudios realizados en España, el primero arroja resultados obtenidos por la aplicación de dos instrumentos de recopilación informativa, uno que evaluó la eficiencia y otro las fortalezas a 454 docentes, según (Herrera-Torres et al. 2019), evidencian que para lograr un trabajo exitoso por parte del profesorado es imperante la contribución en su manejo emocional y cognitivo, por lo que instan a la aplicación de tácticas, procesos y métodos que paralelamente contribuyan en el área profesional y personal.

Una segunda investigación en el mismo país, aplica un análisis estadístico descriptivo y de contingencias a una muestra de 167 docentes, (Vicente-de-Vera-García \& GabariGambarte, 2019), mencionan que cuando la visión de eficiencia laboral en un docente 
tiende a la baja, el cansancio emocional y la pérdida de su individualidad facilitan el desarrollo del síndrome de burnout o del trabajador quemado. Ambos planteamientos consideran el estado emocional como una piedra angular en el desempeño profesional de los docentes.

En otra investigación realizada por (Kim \& Li, 2021), evidencian que la utilización de video bajo la modalidad de narrativa digital refleja principalmente la interiorización de las experiencias, en los participantes, pero también de forma reflexiva logran comunicar las características de su identidad. Aspecto que conlleva a considerar esta estrategia como una opción interesante para procesos de reflexión y mejora.

En el mismo país, pero en otra investigación realizada, los resultados encontrados bajo la metodología cualitativa de análisis de caso aplicada a docentes por (Kim et al. 2021), muestran que el uso de la narrativa digital tiene un impacto en la identidad profesional de los profesores y la generación de emociones positivas sobre la aplicación de su profesionalismo. Dichos productos pueden ser generados de manera individual o grupal. La presente propuesta permitiría abren un abanico de opciones para la producción productos en narrativa digital [audio, videos o híbridos], no solo para producirlos por parte de la gestión directiva, sino también como parte del desarrollo colaborativo entre colegas docentes con un alcance mayor aun al compartir el contenido en redes como YouTube. En Ecuador se han realizado estudios científicos considerando a YouTube como una herramienta de aprendizaje. (Padilla et al. 2020), plantean mediante los resultados obtenidos en un trabajo investigativo mixta apoyada en la observación participante, preguntas, entrevistas, talleres y diarios, que las herramientas de estilo narrativa digital y transmedia se convierten en aliados estratégicos bajo la previa comprensión de su potencial, efectiva presencia en aprendizajes de la informalidad y para finalmente acudir a ellas entendiendo y conociendo como usarlas de manera adecuada.

Así mismo, en un estudio de carácter descriptivo, por medio del análisis documental de manera empírica a docentes de la ciudad de Manta, (Posligua-Anchundia \& Zambrano (2020) concluyen que el uso de la herramienta de YouTube, representa una coyuntura 
de innovación, un aliado para uso de TIC, apoyo en el proceso de enseñanza-aprendizaje y aprovechamiento de oportunidades en su aplicabilidad de formación. Considerando todas estas aristas presentes en los estudios mencionados, se puede comprender, lo interesante y pertinente de la presente investigación para la contribución en el campo científico y educativo.

\section{Narrativa Digital, elaboración de videos y YouTube}

En este sentido, (Hermann-Acosta, 2015), indica que la narrativa digital es un planteamiento sistematizado y apoyado en la tecnología para la elaboración de material de carácter visual, sonoro, textual, estético y animado, que permite plantear de forma creativa e interactiva un producto cuya creación espera realizar un impacto en el receptor. Está completamente relacionada con tres formas de lenguaje: auditivo, visual y sensorial. Estos a su vez llevarán a que diferentes secciones del cerebro sean estimuladas de manera efectiva para una receptividad profunda y significativa de los conocimientos.

Por otro lado, (Kim \& Li, 2021), plantean la narrativa digital como la elaboración de un producto con diferentes herramientas digitales que producirán efectos variados inclusive si su contenido es parecido. La multifuncionalidad de la elaboración, las múltiples opciones de construcción, dispositivos y software o aplicativos enriquecen su ejecución que luego, al ser compartido permite comunicar de manera sincrónica o asincrónica y finalmente, la retroalimentación es manejada por el consumidor. Ambos puntos de vista de los autores dan una clara evidencia de lo práctico, útil y ubicuo del uso de esta técnica y lo recursiva de su producción.

Plataformas como YouTube posibilitan más su alcance y uso según (Ramírez-Ochoa, 2016), dicha red está disponible por medio de suscripción gratuita y permite guardar, gestionar y difundir diferentes tipos de videos. Los productos posteados pueden ser visualizados, descargados, compartidos y hasta viralizados, por usuarios propios y extraños a la página web y en otras redes sociales o plataformas. La cantidad de 
consumidores directos y registrado supera los 100 millones a nivel mundial. A su vez, se ha convertido en una fuente referencial para la formación no formal y ayuda para la educación formal.

En esa misma línea, la investigación de (Padilla et al. 2020), mencionan que dicha plataforma se ha convertido en la fuente común de consulta para efectos académicos 0 de formación. Las comunidades educativas se han volcado a aprender desde la informalidad de este espacio y la ubicuidad que propone, aspecto que permite tener un mayor alcance y contar con un recurso que puede ser revisado las veces necesarias permitiendo un aprendizaje transmedia. Al mismo tiempo representa una posibilidad de generación y creación de contenido con la finalidad de enseñar.

\section{Gestión y fortalecimiento escolar}

A pesar de que el termino gestión escolar representan una disyuntiva académica, (García-Colina et al. 2018), establecen que esta es la concepción del conglomerado de operaciones que se encuentran enfocadas en el alcance de objetivos institucionales en diferentes frentes, que son diseñadas y evaluadas de manera continua. En ese contexto se entiende que tenemos varios personajes que fungen como parte de dicho constructo, pero bajo el liderazgo de quien realiza la labor directiva. Por lo tanto, la operatividad, organización, planificación y evaluación son parte de la norma administrativa.

En esa misma línea se comprende que la gestión al ser un proceso, debe ser fortalecida de manera continua. Ante ello, (Atencio-Bravo et al. 2019), aseguran que el gestor directivo debe considerar el desarrollo de pertenencia a los integrantes de la institución, aprecio por la diversidad e individualidad, respeto por el contingente humano, sus fortalezas en y debilidades en las áreas emotivas, cognitivas y de motivación. Solo esa consideración integral de variabilidad en el equipo y de riquezas estructural podría garantizar resultados efectivos y el alcance de los objetivos trazados para trascender en educación. 
Ambos autores concuerdan en la importancia de la búsqueda de estrategias que permitan generar espacios para que los docentes puedan ser fortalecidos continuamente en su labor como profesionales, formadores y seres humanos, pues el resultado de esa gestión directiva se evidenciará en el trabajo que ejecutan en lo administrativo y lo académico. Por lo tanto, para un eficiente alcance se debe considerar sus individualidades como personas y necesidades colectivas e integrales.

\section{Motivación docente}

El quehacer docente y su eficacia profesional va ligada a su estado motivacional ante su labor, según (Herrera-Torres et al. 2019), debe ser consciente de su estado emocional y sus competencias respecto al manejo de las mismas; ser diestro en su automotivación y lograr conexión empática con los estudiantes, representantes y compañeros de trabajo; y poseer cierta habilidad socio relacional que les permita generar amistades y conservarlas. Por lo tanto, es obvio que la función docente se construye por medio de la integralidad de la persona que ejerce la profesión y no es un evento aislado.

Por otro lado, (Valenzuela et al. 2015), rescata que, desde su preparación inicial, el profesorado debe contar con una formación motivacional, pues no es suficiente con exigírselo en el momento de su labor, ni tampoco con su propio don de gentes; al contrario, es imperante conocer y teorizar la práctica motivacional por parte del profesorado para su ejecución en el día a día de su trabajo. Y como consecuencia, esto se logra al plantear y generar prototipos de aplicación motivacional que favorezcan, afirmen y provoquen su ejecución.

\section{METODOLOGÍA}

Se planteó un enfoque cualitativo, el cual permite la comprensión de acontecimientos, por medio de una exploración a los participantes y su área contextualizada. Este tipo de enfoque proporciona al investigador información pertinente ante las percepciones, experiencias, puntos de vista, interpretación y conceptualización de los fenómenos. La 
recolección de datos se ejecutó bajo la consideración de una cohorte transversal y la técnica utilizada fue el grupo focal (Hamui \& Varela, 2013), definen al grupo focale como un punto de reunión para captar opiniones, sentimientos, percepciones, pensamientos y experiencias de los participantes, para levantar información.

En esa línea, se aprovechó la organización institucional donde se ejecutó una de sus reuniones llamados "laboratorios de ética". El instrumento usado para el levantamiento de datos fue la entrevista de grupo focal a una población de 16 profesores, es decir, todos los docentes. Para el análisis posterior se organizaron los datos en una tabla de segmentación que permita realizar un análisis de los hallazgos.

\section{RESULTADOS}

Los resultados obtenidos en esta investigación por medio de la entrevista de grupo focal enriquecieron la gama de consideraciones que deben existir dentro del ámbito de la motivación de los docentes en su labor diaria. La subjetividad, estados de ánimo y las emociones se evidenciaron como común denominador en todas las respuestas, por lo tanto, los procesos de reflexión en su labor y el generar espacios para compartir sus experiencias son notoriamente importantes para retroalimentar a otros colegas.

Tabla 1.

Análisis entrevista grupo focal motivación docente.

\begin{tabular}{|c|c|c|}
\hline Unidad de análisis & Categorías & Segmento \\
\hline \multirow{7}{*}{$\begin{array}{c}\text { Estado } \\
\text { motivacional/emocional }\end{array}$} & \multirow{6}{*}{ Motivación ante su profesión } & Educar para la vida \\
\hline & & Vocación real por enseñar \\
\hline & & Formar buenos seres humanos \\
\hline & & Influenciar en proyectos de vida \\
\hline & & Agente de cambio social \\
\hline & & $\begin{array}{l}\text { Cambiar el mundo desde la } \\
\text { educación }\end{array}$ \\
\hline & Días más motivantes & Viernes \\
\hline
\end{tabular}


Revista Arbitrada Interdisciplinaria KOINONIA

Año VI. Vol VI. N4. Edición Especial: Educación III. 2021

Hecho el depósito de Ley: FA2016000010 ISSN: 2542-3088

FUNDACIÓN KOINONIA (F.K). Santa Ana de Coro. Venezuela.

Jener Fabricio Chica-Correa; María Isabel Álvarez-Lozano; Claudio Fernando Guevara-Vizcaíno

Miércoles

Lunes

Gratitud de los actores educativos

Resultados de cambio actitudinal

Motivación extrínseca

en los aprendices

Resultados en el rendimiento académico

Recordar el propósito de mi

Manejo de emociones y Condiciones que permiten

profesión

Automotivación

el manejo de emociones

Reflexionar sobre lo que hago

Poner en mi pensamiento los

rostros de mis estudiantes

Escuchar atentamente

Ser observador/a a los cambios en

las personas

Habilidad socio

Aplicación de habilidades

Preocuparse por su estado

relacional

socio-relacionales y empatía

emocional

Ponerse en el lugar de los demás

General relaciones que duren con todos los actores educativos

Llegar a acuerdo en las diferencias

Fuente: Entrevistas.

En el apartado de estado emocional y motivacional, categoría motivación ante su profesión, queda demostrado que hay un compromiso en la formación de los estudiantes para la vida, además la influencia en su labor docente normada por su vocación, el anhelo de formar buenos seres humanos, considerarse entes de influencia para los proyectos de vida de los estudiantes, auto percibirse como agentes de cambio y el deseo utópico de querer cambiar el mundo desde el frente educativo. Sin embargo, el estado motivacional tiende a variar dependiendo de los días de la semana que se cursa, los más motivantes son los viernes, miércoles y lunes. La categoría, motivación extrínseca, permitió ver que recibir gratitud de los actores educativos, ver cambios positivos en la actitud de los aprendices y evidenciar resultados en el rendimiento académico son fuente de motivación para los docentes. 
En la unidad sobre el manejo de las emociones y automotivación, categoría condiciones que permiten el manejo de las emociones, se observó que recordar el propósito por el cual se ejerce la profesión, reflexionar sobre lo que hace y tener presente a sus estudiantes en su memoria como fuente de inspiración es plenamente influyente para autogenerar contención emocional.

Finalmente, en habilidad socioemocional, categoría aplicación de habilidades sociorelacionales y empatía, permitió entrever que la escucha atenta, ser testigos oculares de cambios de las personas, preocuparse por su situación emocional, ponerse en el lugar de los otros (empatía), generar y construir relaciones de valor y duraderas y llegar acuerdos cuando has diferencias, también fomentan motivación en los docentes.

\section{PROPUESTA}

El proyecto de gestión escolar "Ethos-Pro" se plantea considerando 5 pasos a ejecutarse de forma específica y clara: reflexión, concreción (escritura), elaboración, contribución (compartir) y distribución de las experiencias de los docentes para influenciar en su estado emocional y motivación de su proceder profesional.

\section{ETHOS-Pro}

PROYECTO DE GESTIÓN ESCOLAR

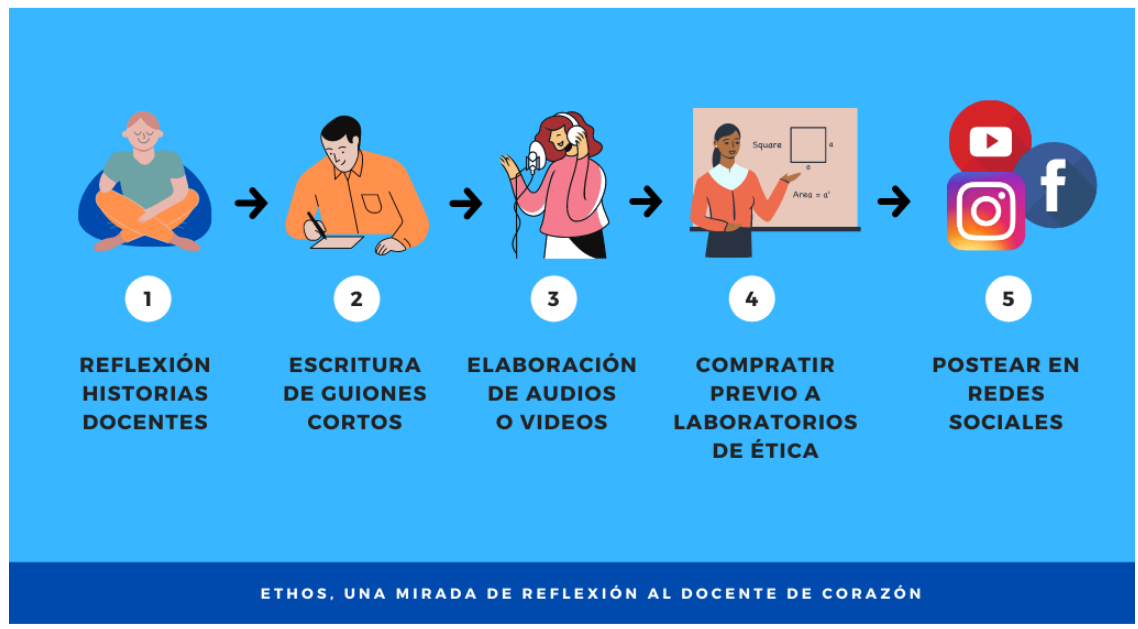


Figura 1. Proceso ETHOS-Pro. Elaboración: Los autores.

A continuación, se detalla cada apartado de la propuesta:

Reflexión de historia docentes: se plantea el que los docentes piensen y reflexiones sobre el impacto de su labor, misma que según sus propias concepciones van más allá de un ejercer una vida profesional y se convierte en encuentros con otros seres humanos a quienes se influencia. Durante un mes se da el espacio en los grupos y áreas de trabajo para que conversen sobre sucesos que alimentan su espíritu vocacional.

Escritura de guiones cortos: durante esos tiempos de conversación y diálogo docente se motiva a la escritura de guiones cortos sobre dichos acontecimientos, establecer personajes que han intervenido en dichas experiencias y recuerdos y en los cuales deje una lección de vida y reflexión de dicho acontecimiento.

Elaboración de audios o videos: para pasar de la documentación a la generación de contenido se propone evidenciar dicho guion con el uso de herramientas digitales que permitan aumentar la carga emocional de los mismos. Es decir, generar un video o audio [narrativa digital] con fondos musicales, imágenes, efectos, etc., que lleven a la ambientación e implicación de los futuros receptores de dicho contenido.

Compartir previo a iniciar sus laboratorios de ética: para la evaluación del impacto, se compartirá en los "laboratorios de ética institucional" espacios de profesionalización, círculos de estudio y acompañamiento pedagógico, que tienen los docentes con la autoridad. El producto no se evaluará con rangos aceptación si es correcto o no, sino que servirá para retroalimentar sobre el impacto emocional y motivacional en los colegas. 
Postear en redes sociales (YouTube): finalmente, los productos serán compartidos en la red social YouTube con la que cuenta la institución para compartirlos con los otros actores educativos e inclusive con colegas de otras instituciones para aumentar su alcance de reflexión a la labor de la enseñanza y aprendizaje, pero con un enfoque de formación integral de ser humanos, conforme los hallazgos evidenciados.

\section{CONCLUSIONES}

La motivación en los docentes es un pilar fundamental como herramienta de contención emocional para su gestión profesional y he ahí la importancia de usar estrategias que ayuden a contar con espacios físicos y virtuales que procuren un reflexionar continuo, y como consecuencia se vea inspirado e impulsado, desde su vocación, su labor como formadores de vidas.

La gestión escolar que ejerce el directivo en su plan de acompañamiento pedagógico debe considerar, no solo la formación continua en el área profesional, sino también dichas actividades propuestas deben estar enfocadas de manera estratégica en la motivación y reflexión como proceso de cuidado integral al educador para la efectividad de la enseñanza y su influencia en el aprendizaje.

La gestión educativa debe estar orientada a mejorar el quehacer y desempeño docente, puesto que al mejorar estos parámetros implícitamente se mejora la calidad en los centros educativos y con eso la consecución de aprendizajes significativos en los estudiantes.

El fortalecer quehacer docente es fundamental en pro de alcanzar la calidad educativa, en este sentido las narrativas digitales toman un papel preponderante para potenciar la labor del profesor en el aula, por la motivación implícita que coadyuva a un mejor desenvolvimiento de las clases.

La narrativa digital, previo a espacios de generación de productos y contenido, fruto de la reflexión concienzuda y provocada dentro de la institución, evidencia, revaloriza y motiva a conocer experiencias de los docentes. Su alcance trasciende a espacio físico y 
puede ser usado y reutilizado por la misma institución y otras instituciones como herramienta para el bienestar de la comunidad educativa.

\section{FINANCIAMIENTO}

No monetario.

\section{AGRADECIMIENTO}

A la Universidad Católica de Cuenca; por todo el apoyo brindado en la motivación y desarrollo de esta investigación.

\section{REFERENCIAS CONSULTADAS}

Atencio-Bravo, E. A., Ramírez Lora, L. J., \& Zappa Berastegui, Y. B. (2019). Neuroliderazgo como estrategia para el fortalecimiento de la gestión directiva en instituciones educativas [Neuroleadership as a strategy for strengthening of directive managment at schools]. Actualidades Investigativas en Educación, 20(1), 29. https://n9.cl/ptj4y

Calderón, L. C., Londoño, O. L., \& Maldonado, L. F. (2014). Guía para construir estados del arte [Guide for states of the art's construction]. http://repositorio.minedu.gob.pe/handle/123456789/4637

García-Colina, F. J., Juárez Hernández, S. C., \& Salgado García, L. (2018). Gestión escolar y calidad educativa [School managment and Educational quality]. Revista Cubana de Educación Superior, 37(2), 206-216.

Hamui, A., \& Varela, M. (2013). La técnica de grupos focales Investigación en Educación Médica [Focus groups' technique investigation in medical education]. Investigación en educación médica, 2(5), 55-60.

Hermann-Acosta, A. (2015). Narrativas digitales como didácticas y estrategias de aprendizaje en los procesos de asimilación y retención del conocimiento [Digital storytelling as didactics and estrategies into the process of assimilation and retention of knowledge ]. Sophía, 1(19), 253. https://doi.org/10.17163/soph.n19.2015.12 
Revista Arbitrada Interdisciplinaria KOINONIA

Año VI. Vol VI. N4. Edición Especial: Educación III. 2021

Hecho el depósito de Ley: FA2016000010

ISSN: 2542-3088

FUNDACIÓN KOINONIA (F.K). Santa Ana de Coro. Venezuela.

Jener Fabricio Chica-Correa; María Isabel Álvarez-Lozano; Claudio Fernando Guevara-Vizcaíno

Herrera-Torres, L., Perandones González, T. M., \& Sánchez-Sánchez, L. D. C. (2019). Fortalezas personales y eficacia docente [Personal strength and teachers effectiveness]. International Journal of Developmental and Educational Psychology. Revista INFAD de Psicología., 317. https://doi.org/10.17060/ijodaep.2019.n1.v1.1431

Katz, I., \& Shahar, B.-H. (2015). What makes a motivating teacher? Teachers' motivation and beliefs as predictors of their autonomy-supportive style. School Psychology International, 36(6), 575-588. https://doi.org/10.1177/0143034315609969

Kim, D., \& Li, M. (2021). Digital storytelling: facilitating learning and identity development. J. Comput. Educ. 8, 33-61. https://doi.org/10.1007/s40692-020-00170-9

Kim, D., Long, Y., Zhao, Y., Zhou, S., \& Alexander, J. (2021). Teacher professional identity development through digital stories. Computers and Education, 162(October), 104040. https://doi.org/10.1016/i.compedu.2020.104040

Ley Orgánica de Educación Intercultural [LOEl], (2011). https://n9.cl/1fo3

Padilla, E. J., Portilla, G. I., \& Torres, M. (2020). Autonomous learning and digital platforms: use of YouTube tutorials of young people in Ecuador [Aprendizaje autónomo y plataformas digitales: El uso de tutoriales de YouTube en jóvenes de Ecuador]. Estudios pedagógicos (Valdivia), 46(2), 285297. https://dx.doi.org/10.4067/S0718-07052020000200285

Posligua-Anchundia, R., \& Zambrano, L. (2020). El empleo del Youtube como herramienta de aprendizaje [Using YouTube as a learning tool]. ReHuSo:Revista de ciencias humanísticas y sociales, 5(1),10-18. https://n9.cl/lbusd

Ramírez-Ochoa, M. (2016). Posibilidades del uso educativo de Youtube [Possibilities of the educational use of YouTube]. Ra Ximhai, 12(6),537-546. https://www.redalyc.org/pdf/461/46148194036.pdf

Reglamento General a la Ley Orgánica de Educación Intercultural [R-LOEl], (2016). https://n9.cl/bca5

Valenzuela, J., Valenzuela, C. M., Silva-Peña, I., Nocetti, V. G., \& Gandarillas, A. P. (2015). School motivation: Keys to future teachers' motivational training [Motivación escolar: Claves para la formación motivacional de futuros docentes]. Estudios Pedagogicos, 41(1), 351-361. 
Revista Arbitrada Interdisciplinaria KOINONIA

Año VI. Vol VI. N4. Edición Especial: Educación III. 2021

Hecho el depósito de Ley: FA2016000010 ISSN: 2542-3088

FUNDACIÓN KOINONIA (F.K). Santa Ana de Coro. Venezuela.

Jener Fabricio Chica-Correa; María Isabel Álvarez-Lozano; Claudio Fernando Guevara-Vizcaíno

Vicente-de-Vera-García, M. I., \& Gabari-Gambarte, M. I. (2019). Niveles de burnout en docentes de secundaria: un estudio descriptivo analítico [Burnout levels in highschool teachers: A descriptive analitic research]. International Journal of Developmental and Educational Psychology. Revista INFAD de Psicología., 2(1), 455. https://doi.org/10.17060/ijodaep.2019.n1.v2.1485

(C2021 por los autores. Este artículo es de acceso abierto y distribuido según los términos y condiciones de la licencia Creative Commons Atribución-NoComercial-Compartirlgual 4.0 Internacional (CC BY-NC-SA 4.0) (https://creativecommons.org/licenses/by-nc-sa/4.0/). 